\title{
Cáncer de colon: del adenoma a la terapéutica endoscópica en oclusión
}

\author{
Rafael Barreto-Zúñiga* \\ Departamento de Endoscopia, Instituto Nacional de Ciencias Médicas y Nutrición Salvador Zubirán, Ciudad de México, México
}

\begin{abstract}
Resumen
La resección endoscópica mucosa (REM) disminuye la incidencia de cáncer colorrectal, las características de la lesión permitirán la estrategia de resección (tamaño, morfología, acceso). La conclusión es que se necesitan revisar las técnicas actuales de REM para obtener mejores resultados, ya que los cánceres de intervalo se relacionan en un porcentaje con resecciones incompletas, aun en pólipos diminutos. REM es reconocida como un tratamiento general para pólipos colorrectales debido a su facilidad y seguridad.
\end{abstract}

Palabras clave: Pólipo. Polipectomía difícil. Resección endoscópica mucosa. Pinza fría. Asa caliente.

La detección de adenomas y su remoción disminuyen la incidencia del cáncer de colon y recto (CCR). La remoción de pólipos aun en lesiones pequeñas no se está haciendo de manera adecuada, por esta razón se presenta el concepto de "polipectomía difícil" sin importar el tamaño del pólipo. La controversia de los métodos de resección en DDW 2019 continua'. El objetivo principal de la presentación relacionada a resección endoscópica mucosa (REM) en el simposium ASGE-AMEG en la DDW 2019, fue la de mostrar algunos aspectos de REM en colon y recto como práctica endoscópica en centros mexicanos. Las técnicas avanzadas para el tratamiento de lesiones superficiales colorrectales representan tratamientos curativos de mínima invasión necesarios para el endoscopista avanzado. Los pólipos difíciles se definen como aquellas lesiones que no pueden ser removidos por un endoscopista promedio, debido a su tamaño, localización y/o morfología. REM es un tratamiento conocido ampliamente para pólipos colorrectales debido a su simplicidad técnica y baja tasa de efectos colaterales. Muchos estudios en centros especializados han demostrado que la poca experiencia en REM se asocia con resecciones incompletas. La prevalencia de lesiones no polipoides en el INCMNSZ en la Ciudad de México en dos periodos de tiempo, presentan un rango del $9.8 \%(59 / 569)$ con REM en el $41.6 \%$ y el $16 \%$ (en 114 adenomas consecutivos en $2001^{1,2}$. Durante colonoscopia, pólipos pequeños $(6-9 \mathrm{~mm})$. Diminutos $(5 \mathrm{~mm})$ de colon y recto son frecuentemente detectados (60-70\%) y generalmente son removidos con fórceps de biopsia. Actualmente, la técnica de polipectomía utilizada para pólipos diminutos es variable entre gastroenterólogos y endoscopistas y el abordaje depende ampliamente de la experiencia individual. Aunque la polipectomía con pinza fría es simple, los bajos porcentajes de resección completa son bajos e inadecuada para pólipos diminutos. Polipectomía con asa fría (PAF) se recomienda para pólipos pequeños debido a que se alcanzan altos porcentajes de resección endoscópica. Sin embargo, para algunas lesiones no polipoides (planas Ila y

\section{Correspondencia:}


deprimidas, IIc) resulta difícil definir los márgenes de la lesión en el asa y resecar por completo. REM es una terapia de primera línea segura y eficaz para remover en colon lesiones mayores de $20 \mathrm{~mm}$ del tipo lateralmente extendido a través de inyección submucosa (generalmente solución salina teñida con índigo carmín), el pólipo se eleva (un "colchón" mucoso) y el margen se define claramente, el cual permite una zona de seguridad y facilita la resección con asa. En un estudio realizado por el Dr. Shang y Dr. Yao en Shangai el año pasado, concluyen que la REM fue significativamente superior para la resección completa cuando se comparó con la polipectomía con asa fría convencional $(\mathrm{PAF})^{3}$. Polipectomía con asa fría (PAF) es la técnica primaria para resecar pólipos $<10 \mathrm{~mm}$. La técnica es efectiva, eficiente, y elimina los riesgos de sangrado tardío y perforación. Sin embargo, datos relacionados en pólipos con pedículo son escasos. En este estudio retrospectivo multicéntrico en pacientes quienes fueron sometidos PAF en 21,871 colonoscopias con un total de 94 Ips en 93 pacientes, una media en tamaño de Ip $6.2 \pm 1.7 \mathrm{~mm}$ con $43 \%$ en colon derecho (76\% adenomas; $4 \%$ hiperplásicos y otros en el $20 \%$ ) resección completa en el $97 \%$ de Ips. Ningún paciente con efectos colaterales. Los autores concluyen que la resección de Ips con polipectomía con asa fría es segura. Interesante artículo, aunque era de esperarse los resultados debido al tamaño de los pólipos Ips ${ }^{4}$. Por otra parte en un estudio observacional de pacientes con PAF y terapia antitrombótica (excepto warfarina) en pólipos menores de $10 \mathrm{~mm}$ incluyendo pediculados vs con aquellos sin terapia antitrombótica. Un total de 2152 pacientes (67.6 \pm 10.9$)$ y 4,433 pólipos colorrectales solo el $11 \%$ recibía terapia antitrombótica. El porcentaje de sangrado tardío fue mayor en el grupo con terapia antitrombótica (ATT 1.67\%, 4/240 vs control 0.47\%, 9/1912, $p=0.024)$. Hemorragia tardía en PAF es rara (menos 2\%) esto ocurre con más frecuencia en pacientes con ATT aun con cesación temporal, en general es segura excepto en aquellos con pólipos cercanos a los $10 \mathrm{~mm}$. Un estudio japonés con varios sesgos $^{5}$. En un de revisión sistemática de la literatura para la remoción de pólipos $>10 \mathrm{~mm}$ utilizando PAF (en un análisis de 522 pólipos) concluyen que esta técnica parece ser segura y efectiva ya que los porcentajes de sangrado tardío $(0.5 \%)$ recurrencia $(4 \%)$ porcentajes más bajos que aquellos con polipectomía convencional (asa caliente) ${ }^{6}$. El tipo de ajuste en la configuración del electrocauterio que podría ser utilizado en polipectomía ha sido materia de debate, y se desconoce si el tipo de configuración (ajuste) del electrocauterio, afecta la eficacia de resección, riesgo de eventos adversos, o porcentajes de recurrencia. Este estudio multicéntrico aleatorizado compara las características de dos ajustes de electrocauterio (coagulación forzada o endocut; ERBE) para REM de pólipos colorrectales grandes (>20 $\mathrm{mm}$ no pediculados). Un total de 928 pacientes fueron aleatorizados (464 Endocut, 464 coagulación forzada) 919 pacientes completaron 30 días de seguimiento. Este es el primer estudio aleatorizado para evaluar los ajustes de configuración en la resección de pólipos mayores de $20 \mathrm{~mm}$ no pediculados. Se demostraron algunas diferencias técnicas entre el microprocesador controlado Endocut y los grupos de coagulación forzada. Sin embargo, ningún grupo fue superior al otro con respecto a eficacia y seguridad. Por lo tanto, la elección del ajuste de electrocauterio podría basarse en experiencia y preferencia del endoscopista ${ }^{7}$. Se ha evaluado la REM comparado con cirugía para pólipos grandes y complejos, en este estudio en USA evalúan a la REM como primera línea de tratamiento para reducir morbimortalidad comparando con la resección quirúrgica, se incluyeron 306 (REM éxito en el 97\%) y 86 pacientes (cirugía) de manera retrospectiva. La morbilidad demostró una reducción significativa en el grupo de Rem cuando se difirió REM en lugar de cirugía en pólipos grandes o complejos ${ }^{8}$.

\section{Resección de espesor completo en colon y recto}

La resección de espesor completo (full-thickness resection device FTRD) ha sido recientemente incorporada al arsenal de terapias endoscopicas en el mundo, en este estudio multicéntrico llevado a cabo en USA (10/17 y 10/2018), en este estudio retrospectivo se colectaron un total de 79 pacientes ( 65 años con $40 \%$ del sexo femenino) los cuales fueron sometidos a EFTR (por sus siglas en inglés: Endoscopic Full-Thickness Resection), de lesiones colónicas utilizando FTRD, la indicación con más frecuencia fue adenoma difícil (recurrente, sin levantamiento adecuado, localizado en orificio apendicular y/o divertículo). El éxito clinico se logró en 67 pacientes (84.8\%), en 12 pacientes con falla al procedimiento (se efectuó en 5 con técnica en asa caliente, 2 con disección endoscópica submucosa y un con cirugía). Los autores concluyen en este estudio multicéntrico que la EFTR es posible hacer, relativamente segura y efectiva en lesiones colónicas difíciles ${ }^{9}$. 
Manejo de Estenosis. Prótesis colónicas. Existen múltiples reportes relacionados con los beneficios de prótesis autoexpandibles para la paliación de cáncer colorrectal no resecable o como puente antes de la cirugía. Las prótesis presentan un bajo porcentaje de complicaciones con una mejor calidad de vida. Los riesgos de las prótesis incluyen migración por arriba del $11.8 \%$, oclusión en el $12 \%$ y perforación en el $4.5 \%$. Los reportes relacionados con la efectividad de colocar prótesis en colon comparado entre cáncer colorrectal (CCR) primario y lesión extra-intestinal son insuficientes. En este estudio retrospectivo japonés compararon estas variables (Hospital St. Luke del 2011-2018) el número de pacientes con CCR primario y tumor maligno extraintestinal fueron $n=18$ y $n=10$ respectivamente. Los autores concluyeron que no existió diferencia significativa entre ambos grupos (en cuanto a éxito clínico); sin embargo, el pronóstico de sobrevida a largo plazo para aquellos con estenosis extracolónica fue malo ${ }^{11}$. En conclusión, la presente revisión trata de mostrar una breve actualización de los trabajos publicados en forma de resumen o sesión en DDW2019, relacionado en REM y polipectomía (técnicas de asa fría, o resección bajo-agua) y paliación en cáncer colorrectal avanzado.

\section{Conflicto de intereses}

No existen conflictos de interés. Sin patrocinio de la industria.

\section{Bibliografía}

1. Barreto-Zuñiga R, EMR Secrets for Colonic Lesions. Presentation Clinical Symposium ASGE/AMEG (Asociacion Mexicana de Endoscopia Gastrointestinal), in DDW 2019. San Diego Cal. USA: DDW; 2019; Session Number 2040; Sp40.

2. Barreto-Zuñiga R, Lopez E, Bobadilla DJ, Maruyama M, Adenomas Planos en Mexico: estudio de 351 Colonoscopias Consecutivas en el INCMNSZ. Endoscopia 2001:12(3):166.

3. Zhang K, Gao P, Bin H. et al. Polypectomy for complete endoscopic resection of small colorectal polyps. Clinical trial registration number: Hongwei-1102-12. Gastrointest Endosc 2018;87:733-40.

4. Kaltenbach T, Keswani R, Nguyen-Vu T, et al. Cold Snare Polypectomy Appears Safe for the Resection of Small Pedunculated Polyps. Accepted Abstracts DDW 2019 (Abstracts). San Diego Cal. USA: DDW; 2019; Presentation Number Su 1694.

5. Aizawa M, Utano K, Nemoto T, et al. Delayed Hemorrhage After Cold Snare Polypectomy in Patients Receiving Antithrombotic Therapy: An Obervational Study of 2152 Patients. Accepted Abstracts DDW 2019 (Abstracts). San Diego California USA: DDW; 2019; Presentation Number 477.

6. Spadaccini M, Chandrasekar T, Aziz M, et al. Endoscopic Removal of Colorectal Polyps > 10mm Using Cold Snare Resection Techniques: A Systematic Review and Pooled-Analysis of the Published Literature. Accepted DDW 2019. San Diego California USA: 2019; Presentation Number: Su1736.

7. Pohl H, Grimm I, Moyer M, et al. Electrocautery Setting Does Not Affect Efficacy and Safety of Endoscopic Resection of Large Colorectal Polyps. Presentation Number, Accepted Abstracts DDW 2019 (Abstracts). San Diego Cal. USA,: DDW; 2019; Presentation Number Sa 215.

8. Patel M, Haque M, Mutha P, et al. Endoscopic Resection Reduces Morbidity when Compared to surgery as First-Line Strategy for Veterans with Large and Complex Colorectal Polyps. Accepted Abstracts DDW 2019 (Abstracts). San Diego Cal. USA: DDW; 2019; Presentation Number Su 1723.

9. Ichkhanian Y, Vosoughi K, Sharaiha R. et al. Non-Exposure Full-Thickness Resection of Colonic Lesions in the U.S: The FTRD Experience. Accepted Abstracts DDW 2019 (Abstracts Book). San Diego Cal. USA: DDW; 2019; Presentation Number Sa 1532 Checar numero).

10. Kumar A, Kim JL. Colonoscopy: Advanced and Emerging Techniques- A Review of Colonoscopic Approaches to Colorectal Conditions. Clin Colon Rectal Surg 2017;30:136-144.

11. Shiratori Y, Oguri N, Ikeya T. A Comparative Study Between Colonic Stenting for Colonic Stenosis Due to Primary Colorectal Cancer and Extra-Parenteral Malignant Tumor Accepted Abstracts DDW 2019 (Abstracts). San Diego Cal. USA: DDW; 2019; Presentation Number: Mo1651. 